\title{
ACKNOWLEDGEMENT TO REFEREES
}

(C) Springer International Publishing Switzerland 2014

Dear Reader

In this the final issue of CNS Drugs for 2014, the editors and production staff at Adis would like to take the opportunity to look back on all that has been achieved in the last 12 months and to thank the people who have given of their time to ensure we continue to bring you world-class content.

We are pleased to advise you of a number of positive developments involving the Adis journals in 2014. This year saw the journal portfolio increase in size with the transfer of a number of existing Springer journals into the Adis portfolio, as well as the launch of two new open access titles: Drugs - Real World Outcomes targets research involving the use of realworld data to evaluate health outcomes and inform healthcare decision-making, with a particular focus on healthcarerelated 'big data'; and Drug Safety - Case Reports publishes reports of any unintended or adverse effects following administration of a medicinal product, as well as cases involving 'near misses' or a lack of efficacy.

Several of our journals saw healthy gains in the ISI impact factor this year. The biggest gains were made by Molecular Diagnosis and Therapy, which leapt $53 \%$ to 2.589; European Journal of Drug Metabolism and Pharmacokinetics, which increased $39 \%$ to 1.312; American Journal of Clinical Dermatology, which increased $37 \%$ to 2.519; Targeted Oncology, which increased $25 \%$ to 3.458; and The Patient-Patient Centered Outcomes Research, which increased $25 \%$ to 1.957. We look forward to building on these gains in 2015.

The first six titles to be commissioned under the Adis Books programme will have been published by year end and we are developing a robust pipeline of new titles, involving top authors from around the globe. We have appreciated the many topical proposals submitted to date.

As another year draws to a close, we would like to say a big thank you to all the authors who have contributed articles to CNS Drugs. Without their expertise and hard work we would not have been able to publish the journal. The quality of published articles also reflects the significant time and effort put in by the peer reviewers, whose dedication ensures that we continue to publish content of the highest possible standard. In addition to the members of our Honorary Editorial Board, we would like to thank the following individuals who acted as reviewers for articles published in CNS Drugs in 2014:

\author{
Giovanni Addolorato, Italy \\ Neil Aggarwal, USA \\ Laura Airas, Finland \\ Angelo Antonini, Italy \\ Adrià Arboix, Spain \\ Jo Arendt, UK \\ Albert J. Arias, USA \\ Francesc Artigas, Spain \\ David Bakish, Canada \\ Konstantin Balashov, USA \\ Richard Balon, USA \\ Borwin Bandelow, Germany \\ Caroline Barbosa, USA \\ Frank Barone, USA
}

\author{
Michel Baulac, France \\ Martin Beaulieu, Canada \\ Barry J. Bedell, Canada \\ Ettore Beghi, Italy \\ R.H. Belmaker, Israel \\ Martino B. Murri, Italy \\ Christian Benedict, Sweden \\ Sandor Beniczky, Denmark \\ Elinor Ben-Menachem, Sweden \\ David Benton, UK \\ Pratik Bhattacharya, USA \\ Kamilla Blecharz-Klin, Poland \\ Julio Bobes, Spain \\ Robert Boden, Sweden
}


Julien Bogousslavsky, Switzerland

Liliane Borel, France

Mark J. Boschen, Australia

Jorgen G. Bramness, Norway

Gregory M. Brown, Canada

Oscar Bukstein, USA

Roger K. Cady, USA

Jon Campbell, USA

Francisco Campos, Spain

Fabio Caputo, Italy

Richard Carlson, USA

Keri L.H. Carpenter, UK

Jose Carrasco, Spain

Frederick Cassidy, USA

David J. Castle, Australia

Jack J. Chen, USA

A.M. Chronis-Tuscano, USA

Leslie Citrome, USA

Ian A. Clark, Australia

Fredrik Clausen, Sweden

Hannah R. Cock, UK

Manuela Contin, Italy

Jorge Correale, Argentina

Pietro Cortelli, Italy

Marco Cosentino, Italy

James R. Couch Jr, USA

Nancy Covell, USA

Phil Cowen, UK

Patricia K. Coyle, USA

Severine Crettol, Switzerland

Marie Dagonnier, Australia

David J. Daley, UK

Gerrit-Jan de Haan, The Netherlands

Marc de Hert, Belgium

Dominique de Quervain, Switzerland

Sheena Derry, UK

Seymour Diamond, USA

Kai Diederich, Germany

Peter Doshi, USA

Lars Edvinsson, Sweden

Tewodros Eguale, Canada

Hagit Eldar-Finkelman, Israel

Michael Enenbach, USA

Christian Enzinger, Austria

Stanley Fahn, USA

Brent L. Fogel, USA

Susan H. Fox, Canada

Yara D. Fragoso, Brazil

Mark Freedman, Canada

Eija Gaily, Finland

Luca Gallelli, Italy

Serge Gauthier, Canada

S. N. Ghaemi, USA
Antonio Gil-Nagel, Spain

Ragy Girgis, USA

Krystyna Golembiowska, Poland

Juliana Goulardins, Brazil

Anthony Grace, USA

Martin Grosshans, Germany

R. Gurrera, USA

Ekkehard Haen, Germany

Timothy T.C. Hain, USA

Edward D. Hall, USA

Hajo M. Hamer, Germany

Richard A. Hansen, USA

Yvonne M. Hart, UK

Philip D. Harvey, USA

Philip Hazell, Australia

Kerstin Hellwig, Germany

Francois R. Herrmann, Switzerland

Thomas Hillemacher, Germany

Shunsei Hirohata, Japan

Gregory L. Holmes, USA

Samuel F. Hunter, USA

Krista F. Huybrechts, USA

Mari Hysing, Norway

Stuart H. Isaacson, USA

Rakesh Jain, USA

Morgan James, USA

Hye Seon Jeong, Republic of Korea

Russel T. Joffe, USA

Cecilie Johannessen Landmark, Norway

Bankole A. Johnson, USA

Patrick Johnson, USA

Richard S. Jope, USA

Wolfgang H. Jost, Germany

Jon Jureidini, Australia

Siegfried Kasper, Austria

Markku Kaste, Finland

Ben Kearns, UK

Richard S. Keefe, USA

Sidney H. Kennedy, Canada

Bernd C. Kieseier, Germany

Gregory Krauss, USA

Robert L. Kriel, USA

Mikko Kuoppamaki, Finland

Shiao-Lin Lai, Republic of Taiwan

Carlo Lazzaro, Italy

Michel Lecendreux, France

Nicole Lee, Australia

Florence F. Levy, Australia

Alfred Lewy, USA

Volker Limmroth, Germany

Jean-Pierre Lindenmayer, USA

Jennifer L. Lyons, USA

Stephen Mahler, USA 
Gin S. Malhi, Australia

Alice Mao, USA

Pavel Mares, Czech Republic

Colin Martin, UK

Giovanni Martinotti, Italy

Vasilios G. Masdrakis, Greece

Ifigeneia Mavranezouli, UK

Joseph P. McEvoy, USA

Ellen L. McGough, USA

Malachi J. McKenna, Ireland

Mehmet Melikoglu, Turkey

Natasja Menezes, Canada

Juan A. Mico, Spain

Antonio Mirijello, Italy

Stine M. Moen, Norway

Xavier Montalban, Spain

Stuart A. Montgomery, UK

Jaime M. Monti, Uruguay

Thomas Muller, Germany

Jan-Willem Muntjewerff, The Netherlands

Makoto Naoi, Japan

Oliver Neuhaus, Germany

Alexander Neumeister, USA

Jacqueline Nicholas, USA

Jimmi Nielsen, Denmark

Agneta K. Nordberg, Sweden

Peter Nordström, Sweden

John G. Nutt, USA

Dag Nyholm, Sweden

Yu Ohmura, Japan

Jes Olesen, Denmark

M.F. Olive, USA

Celia Oreja-Guevara, Spain

Michael H. Ossipov, USA

Kalpana P. Padala, USA

M.V. Padma Srivastava, India

Chi-Un Pae, Republic of Korea

Emma Palmer, USA

Francesco Panza, Italy

Caroline Papeix, France

Antoine Pariente, France

Natalie Parletta, Australia

Francesco Patti, Italy

Paul P.J. Perry, USA

Ismene, I.L. Petrakis, USA

John S. Phillips, UK

Surasak Phuphanich, USA

Jose Pimentel, Portugal

Emilio Portaccio, Italy

Sheldon H. Preskorn, USA

Chengxuan Qiu, Sweden

Robert R.B. Raffa, USA

Josep A. Ramos-Quiroga, Spain
Mark H. Rapaport, USA

Bappaditya Ray, USA

L. Recht, USA

A.T. Reder, USA

Gary Remington, Canada

Joseph Reoux, USA

Kimberlei A. Richardson, USA

Karl Rickels, USA

Dieter Riemann, Germany

Adelaide S. Robb, USA

Emma S.J. Robinson, UK

Maria A. Rocca, Italy

Felix Rosenow, Germany

Walter Royal, USA

Sean Ruland, USA

Simona Sacco, Italy

Mustafa Sahin, USA

Magnhild Sandberg-Wollheim, Sweden

Jerome Sarris, Australia

Lianne Schmaal, The Netherlands

Johann Sellner, Austria

Richard C. Shelton, USA

Mustafa S. Siddiqui, USA

Werner Sieghart, Austria

Robert Silbergleit, USA

Cesare R. Sirtori, Italy

Edoardo Spina, Italy

Vladan Starcevic, Australia

Hans H. Stassen, Switzerland

Christopher R. Steer, UK

Mark A. Stein, USA

Dai Stephens, UK

J.W. Stewart, USA

Bruno H. Stricker, The Netherlands

$Y$ u-Ping Su, Republic of Taiwan

Craig B. Surman, USA

Takefumi Suzuki, Japan

Michael V. Tatley, New Zealand

Helio A.G. Teive, Brazil

Todd Theile, USA

Dagmar Timmann, Germany

Oivind Torkildsen, Norway

Carlo C. Tornatore, USA

Giuseppe Tosto, USA

Eugen Trinka, Austria

Rudolph Uher, UK

Amber D. Van Laar, USA

Gabriele Vassallo, Italy

Peter Vestergaard, Denmark

Eduard Vieta, Spain

Hugo Vrenken, The Netherlands

Tanya L. Wallace, USA

James Waxmonsky, USA 
Richard H. Weisler, USA

Paul J. Wellman, USA

Gregor K. Wenning, Austria

Michael Westerveld, USA

Andreas A. Westin, Norway

Reinout Wiers, The Netherlands

Hal S. Wortzel, USA

Edwina Wright, Australia

Je Sung You, Republic of Korea
Moussa B.H. Youdim, Israel

Gaetano Zaccara, Italy

Rene P. Zahedi, Germany

Sandro Zambito Marsala, Italy

Suzanna M. Zick, USA

J.M. Zito, USA

Robert Zivadinov, USA

Michele Zoli, Italy

We hope that you have found the articles published throughout 2014 to be both interesting and informative. The editors have appreciated the high quality of content contributed to the journal this year and look forward to keeping you up to date with topical issues in the fields of neurology and psychiatry in 2015.

With best wishes from the staff of CNS Drugs and all at Adis Publications. 